\title{
Synthèse du \\ Colloque « Risques et Résilience des Territoires Apports de la notion de résilience à la gestion des risques » Paris-Marne-la-Vallée, 10 et 11 octobre $2017^{1}$
}

\author{
Jean-Michel GRESILLON
}

Président du Conseil Scientifique du programme « Risques, Décision, Territoires » du Ministère de la Transition Ecologique et Solidaire Animateur du colloque

Les risques naturels et technologiques ont longtemps été abordés sous l'angle de la prévention, de la protection, de l'amélioration de la connaissance de l'aléa à l'origine du risque. Puis, avec l'apparition de la notion de vulnérabilité, la communauté des chercheurs, des ingénieurs et des gestionnaires du risque a élargi son regard pour s'intéresser aux enjeux. Le nouveau point de vue véhiculé par la notion de résilience, centrée sur la capacité de rebond après la survenue d'un bouleversement plutôt que sur l'étendue des dommages, séduit par son caractère positif. Mais, au-delà de cet aspect sympathique, qu'est-ce que cette notion de résilience change dans la façon dont les risques peuvent/doivent être abordés et gérés ? Telle est la question initiale posée par ce colloque qui était organisé conjointement par la Société Hydrotechnique de France (SHF) et par le Ministère de la Transition Ecologique et Solidaire (MTES).

L'occasion en a été donnée par la restitution des recherches effectuées dans le cadre de l'appel à proposition de recherche intitulé « La résilience des territoires face aux risques » du programme « Risques, Décision, Territoires », piloté par la Direction de la Recherche du MTES. Le ministère a souhaité ouvrir les débats sur cette notion de résilience et sur son apport à la gestion des risques à l'ensemble de la communauté des chercheurs et des gestionnaires du risque. La SHF, sensible aux apports des Sciences Humaines et Sociales - qui ont joué un rôle majeur dans l'émergence de la notion de résilience pour les risques - $s$ 'est associée à cette démarche.

Le titre du colloque "Risques et résilience des territoires 2017. Apports de la notion de résilience à la gestion des risques » marque bien la rencontre des objectifs communs aux deux organismes :

- faire le point sur cette notion de résilience, sur sa signification à la fois théorique et pratique, sur l'échelle territoriale qu'elle induit, et sur son caractère opérationnel. En bref, il s'agit de s'interroger sur ses apports dans le cadre de la gestion des risques ;

- associer chercheurs de toutes les disciplines, ingénieurs et gestionnaires du risque pour traiter de cette question. Il est en effet essentiel à la fois de faciliter le débat entre les communautés scientifiques : sciences humaines, sciences de la terre, sciences de l'ingénieur, et aussi de sortir la discussion du strict domaine de la recherche et de mettre la résilience à l'épreuve des faits.

22 communications et 9 posters ont été présentés au colloque qui comptait 104 participants. La moitié des communications orales était issue du programme Risques, Décision, Territoires du MTES.

1. http://www.shf-hydro.org/213-1-_risques_et_resilience_des_territoires apport_de_la_notion_de_resilience_rdt- $-71 . \mathrm{html}$
Parmi les participants, 4 personnes venaient de l'étranger ( 2 d'Algérie, 1 de Tunisie et 1 du Canada). L'assistance était composée à $45 \%$ de chercheurs et d'universitaires ; à $23 \%$ de représentants des services de l'état; à $13 \%$ de professionnels privés (Bureaux d'Etudes, architectes...) ; à $9 \%$ de représentants des collectivités et autant d'Associations.

Bien que le thème du colloque soit ouvert à tous les types de risques, la plupart des communications (14 sur les 22) traitaient de risques associés aux risques naturels, et singulièrement aux inondations (12). Les autres envisageaient des risques technologiques (4 sur les 22) ou bien tous les types de risques (4 également). Le thème du changement climatique était explicitement présent dans trois communications orales.

Afin de faire ressortir l'essentiel des idées échangées à l'occasion de ce colloque, la restitution suivante ne respecte pas exactement l'organisation des sessions, ni l'ordre des présentations. Elle s'organise selon les paragraphes suivants qui permettent de rendre compte des idées fortes portées par le colloque :

1) Complexité, polysémie, ambiguïtés de la notion de résilience appliquée aux risques

2) l'indispensable échelle du territoire

3) Aide à la décision et résilience

4) Résilience, concertation, gouvernance

5) Table ronde

6) Conclusions

\section{COMPLEXITÉ, POLYSÉMIE, AMBIGUÏTÉS DE LA NOTION DE RÉSILIENCE APPLIQUÉE AUX RISQUES :}

Dès l'ouverture du colloque Magali Reghezza a rappelé la complexité, la polysémie de la notion de résilience appliquée aux risques : un "mot valise » qui, faisant référence à la capacité d'un système soumis à une perturbation à s'adapter en résistant ou en changeant, renvoie à tous les éléments du « système » (résilience de quoi ? et à quoi ?), à une diversité de manières de rebondir (retour à quel équilibre ?). Qui renvoie aussi à un « arc-en-ciel de sens » (D. Provitolo), à une « notion fleuve » véhiculant un objectif de résultat à atteindre, à moins que ce soit à un processus. Enfin, une notion plus ou moins imposée, comme le suggère le titre de la conférence de M. Reghezza : «La résilience, du mot valise à l'injonction, une notion fleuve à l'épreuve de son opérationnalité ». En dépit de cette complexité et de ces ambiguïtés, la conférencière met en valeur la notion en montrant qu'elle peut servir de guide aussi bien pour préparer la crise, que pour la gérer ou pour aider au relèvement après la crise. Elle souligne son caractère intégrateur qui oblige à penser la crise dans son 
ensemble et non comme à des phases indépendantes les unes des autres. En dépit des difficultés sémantiques signalées, la conférencière présente donc la notion de résilience comme positive pour la gestion des risques.

La première communication, présentée par Céline Cholez (Univ. Grenoble) et Laurence Creton-Cazenave (Paris tech) (« La Résilience, un objet frontière au-delà des risques »), rend compte d'un travail collectif qui a réuni des " académiques », des experts et des professionnels de la gestion des risques pour s'interroger sur le sens de la notion de résilience dans les risques et sur sa mise en œuvre opérationnelle. Ce groupe d'experts confirme le point de vue exprimé par la première conférencière : bien que "venue d'en haut", dans un contexte de réduction de l'engagement de l'Etat, la notion est intéressante en ce qu'elle oblige à réfléchir aux événements perturbateurs, aux ramifications entre leurs causes et leurs effets, aux interactions d'échelles temporelles et spatiales, à la complexité des systèmes impactés. L'auteure pointe une question qui reviendra dans plusieurs communications : puisqu'il s'agit de se relever après une crise, que faut-il préserver ? Qu'est-ce qui compte pour un territoire ? Au travers d'un exemple (l'aménagement du quartier des Ardoines), elle montre que la résilience provient d'une démarche d'apprentissage continu, d'une forme de gouvernance transversale, permettant une meilleure articulation des différents points de vue, des différentes temporalités.

Sur la base d'une enquête sociologique par entretiens « compréhensifs » auprès d'élus et d'acteurs de la gestion des risques dans trois communes de France ayant subi des inondations, l'équipe du Cerema conduite par Marie-Aude Corbillé ("Appropriation des notions de vulnérabilité et de résilience : enseignements de trois territoires inondables ») montre que la notion de résilience n'est pas bien connue, qu'elle est vécue par certains comme une injonction, qu'elle suscite même une certaine méfiance. Pourtant les populations sinistrées savent exprimer les conditions qui, selon eux, pourraient rendre leur territoire plus résilient : elles expriment le besoin de cohérence entre tous les échelons de l'action, d'information et de reconnaissance des traumatismes et des pertes par la société locale, etc.

En montrant la difficulté de définir une mesure pertinente de la résilience, Samuel Rufat (Univ. Cergy Pontoise) («Individus, Sociétés, Territoires : comment mesurer leur(s) résilience(s) ? ») se montre très critique vis-à-vis de la notion elle-même qui suppose un consensus sur ce qu'est le bon fonctionnement d'un territoire : la résilience pour qui ? Dans quels territoires ? Pour quels horizons temporels ? Avec quelle gouvernance ? Pour lui, l'absence de robustesse et de possibilité de mesure de la notion signifie qu'elle est manipulée, qu'elle donne lieu à des discours qu'il faut déconstruire, que son emploi met en œuvre des arbitrages implicites qui éludent les questions essentielles pour les populations.

Bien entendu, les questions posées aux conférenciers portent surtout sur le paradoxe mis en évidence par presque toutes les communications précédentes : la résilience : une notion utile pour son caractère intégrateur ? Ou bien un leurre, une notion mal définie et résultant d'une injonction de l'Etat liée à son désengagement ? Un outil à saisir pour réfléchir à ce qu'il faut préserver sur chaque territoire ou une création d'inspiration néolibérale et peu démocratique ? A ce stade, les débats conduisent, hélas, à des échanges un peu décevants où une forme d'enthousiasme pour la notion, clairement affichée pourtant par les orateurs, n'ose plus s'exprimer face au doute - un peu dogmatique - exprimé sur son origine (et qui s'est avéré plus apte à fermer les questions qu'à les ouvrir ?).

\section{L'INDISPENSABLE ÉCHELLE DU «TERRITOIRE »}

L'une des vertus de la notion de résilience est d'obliger à penser les risques à l'échelle du territoire susceptible de les subir. Sont présentées ci-dessous les communications qui illustrent le rôle central de cet aspect territorial impliquant la prise en compte de toutes les dimensions des territoires, spatiales, temporelles (historiques), mais aussi économiques et sociales, morphologiques, démographiques, etc. Dans ces communications, la résilience apparaît comme une façon de réfléchir au territoire avant de parler du risque. Le risque est une composante du territoire et il s'agit de réfléchir au premier en tenant compte de l'autre.

Le travail présenté par Virginie Duvat (Univ. La Rochelle): "Reconstruire les chaînes d'impact des événements passés pour renforcer la résilience des territoires " concerne les effets des événements météo-marins (cyclones, tempêtes) sur un type de territoire particulier : les atolls en Polynésie française. Au moyen d'une recherche documentaire, de photographies aériennes et d'entretiens auprès des populations qui ont subi les cyclones, les effets de ces derniers sont reconstitués et analysés afin de définir leurs impacts emboités sur les différentes composantes du territoire de trois atolls voisins : morphologie, écosystèmes, économie, démographie. La reconstitution des " chaînes d'impact » intègre les dimensions spatiales et temporelles des atolls et conduit à définir des pistes pour renforcer la résilience de ces territoires fragiles, notamment au travers d'une meilleure gestion des cyclones. V. Duvat note au passage que les cyclones peuvent avoir des aspects bénéfiques (apports de sédiments qui exhaussent l'île localement).

Avec "Enjeux de l'intégration des espaces naturels littoraux dans la gestion des risques liés à la mer ", son collègue Camille Salmon (Univ. La Rochelle) s'intéressait à une problématique voisine, celle de la fonction de protection par rapport aux cyclones des espaces naturels sur les littoraux des îles de Tubuai en Polynésie française et de la Réunion. Par une analyse diachronique des littoraux de ces deux iles sur des périodes de 35 à 60 années, l'effet protecteur des «zones tampon » situées entre le pied de la plage et les premiers enjeux bâtis est montré. La restauration de ces zones naturelles apparait comme une condition pour réduire les risques et améliorer la protection contre les événements marins violents que subissent ces territoires.

Céline Debrieu-Levrat (Conseil départ. De la Gironde) présentait une communication intitulée "la résilience territoriale : enjeux, acteurs et stratégies en Dordogne girondine ». Il s'agit d'un travail destiné à anticiper le développement du territoire girondin, notamment dans ses Territoires à Risque d'Inondation. Pour ce faire, une démarche d'analyse historique des stratégies individuelles et collectives adoptées dans les communes inondables du département est en cours. Elle devrait permettre d'approfondir la compréhension locale du système eau/territoire et conduire, via l'élaboration de critères de résilience pour les différents espaces naturels et urbains, à une traduction opérationnelle de la résilience dans les planifications territoriales futures (SCOT, PLU ...).

Les territoires sur lesquels Annabelle Moatty (Univ. Montpellier) et ses collègues ont travaillé sont des communes du Sud de la France (dans le Var et l'Aude) qu'ils ont étudiées quatre années ou quinze années après qu'elles aient subi une inondation importante. Leur objectif était de savoir si ces communes ont su tirer profit de leur reconstruction pour s'adapter et prévenir le risque d'inondation. L'exposé d'A. Moatty ("Intégrer une éthique préventive 
dans le processus de relèvement post-catastrophe ") développe une analyse solide, appuyée sur des archives et sur des entretiens. Le bilan des reconstructions est mitigé, avec des succès qu'ils attribuent à une solidarité, une connaissance du territoire, une communication réussie entre les acteurs et, inversement, à des échecs liés à des conflits d'intérêts, des contraintes réglementaires, et à une définition défaillante des rôles et des responsabilités.

L'échelle territoriale explorée par l'équipe de Fabien Rival (Cerema) est celle du « quartier». Ils intitulent leur exposé : "Quartiers résilients aux inondations. Quand la résilience vient questionner la prévention en partant du niveau local ». Partant de l'idée qu'il est nécessaire de mieux articuler l'action publique et les solidarités spontanées en cas d'inondation majeure, ils explorent l'hypothèse du quartier comme échelle de mobilisation de ressources, permettant d'intégrer des spécificités très localisées. Ils proposent même une définition de la résilience comme une capacité d'organisation collective en partant de l'échelle du quartier. L'hypothèse formulée est difficile à confirmer mais des pistes sont proposées pour la faire avancer et la tester.

C'est au sujet du territoire du bassin de Lacq, un territoire qu'ils montrent « sous tension » en raison de son histoire industrielle récente et en mutation rapide, que Christine Bouisset (Pau), Sylvie Clarimont (Pau) et Julien Rebottier (cnrs Toulouse) ("L'approche territoriale : Une condition de l'apport de la résilience à la prévention des désastres. Exemple du bassin industriel de Lacq face à ses futurs incertains ») se sont interrogés par une démarche d'enquêtes, d'analyse de documents et d'archives, de revue de presse. Ils montrent que la "vocation industrielle » du territoire est construite, qu'elle se heurte à d'autres regards difficilement pris en compte. Dans ce contexte de pluralité d'acteurs peu reconnue et de tensions sur ce qui importe sur le territoire, la résilience apparait comme une notion peu significative pour la prévention des catastrophes et charriant peu de sens. Ils proposent, dans ce cas, de réduire la résilience à « la possibilité de prendre la moins mauvaise des options pour le plus grand nombre d'acteurs (dont la voix compte?) ».

Le territoire étudié par Christelle Morel-Journel (Univ. Lyon) et al. s'est avéré également rétif au qualificatif de "résilient». Son travail, qui concerne la région de Saint-Etienne, est intitulé «La pollution industrielle des sols en héritage. De l'indifférence à la résilience? ». L'auteure montre que, en s'effondrant dans les années 50, l'industrie minière de la région stéphanoise a laissé en place des sous-sols dégradés, longtemps restés oubliés. A la longue indifférence aux sols pollués fait suite désormais une prise de conscience progressive liée aux coûts induits par ces pollutions sur les opérations d'aménagement des zones concernées. Les auteurs s'interrogent sur le sens que peut revêtir la notion de résilience dans ce contexte où l'accommodement $\mathrm{a}$ longtemps constitué la seule réponse à la pollution des sols.

Une autre dimension territoriale, celle de la ville, a été très présente dans ce colloque. Elle était introduite par la conférence donnée par Sébastien Maire, Haut Responsable de la Résilience pour la ville de Paris. Paris fait partie du réseau des 100 villes résilientes (100 RC) créé par la Fondation Rockefeller pour aider les villes du monde à devenir plus résilientes face aux grands chocs et aux stress qu'elles affrontent au quotidien. La stratégie de résilience de la ville de Paris venant d'être rendue publique, S. Maire nous en a fait le commentaire. De façon remarquable, les premiers enjeux prioritaires de résilience affichés par la ville de Paris sont : 1) les inégalités sociales, économiques, territoriales et la cohésion sociale ; 2) Le risque terroriste et le contexte sécuritaire. Ne viennent qu'ensuite : 3) Le dérèglement climatique ; 4) la pollution de l'air ; 5) la Seine et les risques liés au fleuve ; 6) la gouvernance territoriale. La résilience est ainsi clairement affichée comme liée à une cohésion sociale que les inégalités et l'insécurité compromettent. Cette dimension sociale de la résilience affichée par la ville de Paris illustre clairement l'ouverture que la notion de résilience introduit dans la question des risques.

D'autres communications ont eu pour objet d'étude le territoire urbain : celle présentée par Yona Jebrak, de l'Université du Québec à Montréal se situait également dans le cadre des 100 villes résilientes : "La résilience en question : La déclinaison locale du concept à travers les plans stratégiques 100 Resilient Cities de Christchurch, Nouvelle-Zélande ». Celles de Sylvain Rode "Les transactions entre la ville et l'inondation pour un urbanisme plus résilient ", de Mathieu Bonnefond «La modélisation hydraulique comme condition de la résilience des projets d'aménagement urbain en zone inondable? ", Enfin celle de Hélène Barrière "La définition de la Stratégie Locale de Gestion du Risque Inondation : une opportunité pour développer un processus de résilience de la métropole toulousaine " ont également le territoire urbain comme sujet. Compte tenu des messages qu'elles portent, nous les développerons au paragraphe 4, intitulé Résilience, Concertation, Gouvernance.

Deux posters développaient également une problématique dans laquelle le «territoire » joue un rôle central, ce sont :

Bouameur Oumnia (Univ. De Constantine) : «Le patrimoine culturel : un catalyseur de la résilience des communautés».

Metzger Alexis (Univ. De Strasbourg) : "Être résilient aux sécheresses? Perspectives géo-historiques en alsace $\left(X V I I i^{e}-X^{2} X^{e}\right.$ siècles $) »$.

\section{AIDE À LA DÉCISION ET RÉSILIENCE}

Plusieurs communications ont eu pour objet l'aide à la décision, notamment au travers d'outils techniques. Elles ont pour objectif de contribuer à la résilience par le biais d'outils nouveaux ou d'approches originales qui intéressent un secteur ou un autre de la gestion des risques. Ces communications concernent souvent des actions de réduction de la vulnérabilité ; leurs objets d'étude sont complexes (réseaux, villes, quartiers, entreprises ...), la référence à la notion de résilience tient aussi au caractère systémique des questions traitées ou des approches adoptées.

Cathy Werey (Irstea Strasbourg) a présenté une «Analyse Coûts-bénéfices pour l'évaluation de la résilience des réseaux d'eau potables ». Prenant pour hypothèse une défaillance exceptionnelle (une cyber-attaque) du réseau d'eau potable de la ville de Strasbourg, elle entreprend une Analyse Coûts-Bénéfices (ACB) pour comparer deux scenarios dits « de résilience » par rapport à la situation actuelle prise comme référence. Ces scénarios comportent des coûts d'investissement et de fonctionnement qui sont comparés aux bénéfices qu'ils génèrent et qui sont évalués monétairement par enquêtes sur le consentement à payer des usagers. L'étude devrait constituer un outil d'aide à la décision complémentaire pour les choix d'amélioration du réseau.

Avec "Incertitude et jugement Expert pour l'aide à l'évaluation de la Résilience des réseaux Techniques Urbains » Marc Vuillet (EVP Paris Est) part de l'idée que les gestionnaires des réseaux ont recours aux experts parce qu'ils ont une très mauvaise connaissance des risques de défaillance des infrastructures qu'ils gèrent face à des aléas rares. Le 
travail présenté vise la production de démarches et d'outils scientifiques pertinents pour le recueil et le traitement de dires d'experts fiables ou assortis de niveaux de confiance, dans un contexte d'informations et de données d'entrée incertaines. Il développe une modélisation fonctionnelle pour étudier les effets d'une inondation sur les digues du Drac. Il développe aussi une méthode d'évaluation des dires d'experts. Ce travail vise à objectiver les approches systémiques d'évaluation des risques auxquels ont recours les experts en situation d'urgence.

L'étude «Estimation et amélioration de la résilience d'un territoire aux risques NATECH issus d'une inondation " présentée par Michel Lesbats, de l'université de Bordeaux, et Eric Piatyszek de l'Ecole des Mines de Saint Etienne, vise à améliorer la résilience territoriale au risque Natech (pour Naturel et Technologique) inondation. Elle part de l'hypothèse d'une inondation qui atteint le site industriel du bec d'Ambès, identifie trois zones de fragilité techniques et organisationnelles et cherche à mettre en place un dispositif de réponse graduée des industriels en fonction de la gravité de l'épisode inondant. Par enquête auprès des acteurs, une démarche d'amélioration de l'opérationnalité des dispositifs de maitrise des risques Natech est proposée. La démarche est précisée au travers d'un poster intitulé : "Evénements NATECH inondation - analyse de l'impact sur les sites industriels », présenté par Eric Piatyszek et exposé au colloque.

Avec : "Résilience territoriale : du concept à l'analyse d'infrastructures critiques en montagne ", Jean-Marc Tacnet (Irstea Grenoble) développe une méthode d'analyse de la vulnérabilité des réseaux aux aléas naturels (chutes de blocs, avalanches...). Définissant des indicateurs d'attractivité des points reliés par le réseau (les communes), et d'exposition des segments du même réseau (les routes), il modélise la vulnérabilité indirecte d'un réseau de transport routier pour aider à la décision de mise en place des actions de prévention contre les risques de rupture de segments de routes. Une autre étude sur les infrastructures routières, intitulée "Résilience d'infrastructures critiques face à l'aléa mouvement de terrain : application à l'axe autoroutier A30 » était présentée sous forme de poster par Christian Bouffier (Ineris).

Laurent Millair (Suez Consulting) présentait une communication intitulée "Résilience des usines d'eau potable SUEZ exploitées par Eau du Sud Parisien - leçons tirées de la crue de juin 2016 ». Il y montre que la stratégie déployée pour faire face à l'inondation de l'une des usines au cours de la crue de 2016 a permis d'assurer l'alimentation en eau sans rupture. Toutefois, le retour d'expérience complet de l'événement a permis une ré-expertise de la stratégie et l'élaboration d'outils permettant d'accroitre la résilience des sites de production par rapport au risque d'inondation.

Le sujet traité par Laura Pinson (Ifsttar) concerne une aide à la décision pour un risque émergeant : le risque caniculaire. Sa communication : "Un indice de dangerosité pour une résilience urbaine en période caniculaire » part du constat de l'accélération de la prévalence des périodes caniculaires en France en raison du changement climatique et de l'importance de l'effet d'ilot de chaleur dans les centres villes : elle en déduit la nécessité de définir un indice de dangerosité de ces canicules pour les habitants. L'indice, fondé sur une modélisation des températures adaptée à l'échelle du quartier, tient compte des types d'appartements, de l'importance et de la durée des canicules et de la fragilité des personnes; il devrait permettre une gestion ajustée des canicules ainsi qu'une adaptation des villes au risque caniculaire.
Sur ce thème du changement climatique, plusieurs posters ont été présentés : "Résilience des infrastructures critiques interconnectées aux changements climatiques pour optimiser la gestion des risques (inondation, surcote marine, feu de forêt, événements hydrométéorologiques extrêmes) " présenté par J. Lecroart (Artelia) et : "De la gestion des risques dans les territoires à l'adaptation des territoires aux effets $d u$ changement climatique : une ré-interrogation de la notion de résilience » par Mohamed Taabni (université de Poitiers).

Enfin, le Lieutenant-Colonel Frédéric Castagnola (SDIS Alpes Maritimes) a présenté un exercice de simulation de crise qu'il développe sous le titre de "Module IMPACT : Épreuve de sensibilisation à la conscience du risque et la gestion de crise ». Il s'agit d'une sensibilisation à la gestion des situations d'urgence, avec mises en situation, jeux de rôles et debriefings sous la supervision d'experts en gestion de crise (sapeurs-pompiers, gendarmes ou policiers). Elle est destinée aux Équipes de direction, aux acteurs de la gestion de crise de tous les types de structures susceptibles d'être impactées par des événements déstabilisants.

Quatre autres posters développaient des questions en rapport avec le thème de l'aide à la décision :

Guillaume Prudent-Richard (Artelia) présentait les deux posters : "Résilience du secteur de l'énergie : pratiques émergentes » d'une part, et : "Quel modèles et indicateurs pour mesurer la résilience urbaine?».

Walid Bouchenafa (Université de Technologie de Compiègne) : "Vulnérabilité du métro de Paris aux crues de la Seine ».

Line Kong-A-Siou (BE Mayane) : " Entreprises et foyers résilients face à une inondation, un modèle novateur combinant mesures structurelles et mesures organisationnelles ».

\section{RÉSILIENCE, CONCERTATION, GOUVERNANCE :}

Une autre dimension importante de la résilience est celle de la concertation. La réaction par rapport à un risque ne peut pas être individuelle, elle doit être réfléchie collectivement. Une démarche concertée facilite (sans la garantir) l'acceptation de la gouvernance et constitue un élément clé de la réussite des politiques en matière de risque.

On se souvient que la stratégie de résilience de la ville de Paris, présentée par Sébastien Maire, repose sur l'idée qu'un schéma de résilience ne peut faire consensus que s'il vise la cohésion sociale. On se souvient aussi de celle Bouisset et al. qui, dans le même ordre d'idées, montre la difficulté à faire consensus sur ce qui compte sur un territoire, en l'absence d'une concertation équilibrée. Plusieurs communications illustrent une idée voisine : la concertation entre tous les acteurs est indispensable pour parvenir à des projets de résilience adoptés par les personnes concernées et dont la gouvernance soit acceptée.

La communication d'Hélène Barrière (Toulouse Métropole) et de Jérémy Savatier (ISL) "La définition de la stratégie locale de gestion du risque inondation : une opportunité pour développer un processus de résilience de la Métropole Toulousaine » illustre la démarche collaborative qui a été mise en place pour élaborer cette stratégie locale : enquêtes auprès des 41 communes concernées, création d'un site collaboratif, réunions, ateliers thématiques... ont conduit à la co-construction d'une stratégie et d'une vision de long terme pour la gestion du risque d'inondation à l'échelle de la Métropole qui débouche sur des programmes d'action et de planification urbaine. 
Les deux communications de Sylvain Rode (Univ. Perpignan), Mathilde Gralepois (Univ. Tours), et Eric Daniel-Lacombe (ENS Archi Paris) «Les transactions entre la ville et l'inondation pour un urbanisme plus résilient ", d'une part et de Mathieu Bonnefond «La modélisation hydraulique comme condition de la résilience des projets d'aménagement urbain en zone inondable ? "développent l'une et l'autre la question des projets d'aménagement urbains dans des zones inondables. En prenant pour exemple la réhabilitation de quartiers inondables (notamment dans les villes d'Angers et de Romorantin) ils montrent que ces aménagements urbains atypiques constituent une occasion de dialogue entre les services de l'Etat, les élus locaux et les professionnels du projet urbain. Ils aboutissent, dans le cas de Romorantin par exemple, à des aménagements innovants, certains ayant déjà subi, sans dommages, l'épreuve de l'inondation. Le modèle hydraulique, notamment, est présenté comme un outil facilitant ce dialogue entre professionnels, élus et services de l'état.

En écho à la stratégie de résilience de la ville de Paris, Yona Jébrak (Univ. Québec Montréal) présentait la démarche de la ville de Christchurch dans le même cadre des « 100 Resilient Cities ». Sa communication, intitulée "La résilience en question : La déclinaison locale du concept à travers les plans stratégiques 100 Resilient Cities de Christchurch, Nouvelle-Zélande " rappelle que la ville de Chrischurch a subi deux séismes consécutifs et importants en 2010 et 2011. Le concept de Resilient City conduit à l'idée de modifier le modèle de développement urbain pour le rendre capable de faire face, de s'adapter et de se transformer. La communication oppose la réponse institutionnelle et la réponse citoyenne qui se décline à différentes échelles d'organisations (organisations, communautés, individus...). L'auteur ne cache pas que des conflits de gouvernance ont pu exister au niveau de cette reconstruction.

Avec «Ce que peut apporter la résilience à la prévention des désastres. Exemples en Lavours - Ain (Culoz) et en Chautagne - Savoie (Chanaz) », Patrick Pigeon (Univ. Savoie Mont Blanc) présente un point de vue original et légèrement provocateur : sa présentation illustre le cas de deux communes riveraines situées en bordure du Rhône : Culoz, dans l'Ain et Chanaz en Savoie. Pour des questions de risque résiduel (toutes les deux sont protégées des crues du Rhône par des digues), la première n'a pas été autorisée à ouvrir un port de plaisance le long du Rhône, tandis que la seconde a pu le faire. D'après l'auteur, cette différence de traitement s'explique par le passé de chacune des deux communes et se trouve justifiée au nom de la résilience et du flou que contient la notion. La résilience est ainsi présentée comme une aide à gérer des arbitrages liés aux risques ...

Le poster présenté par Soumaya Bahroun (Univ. St Etienne) mérite également d'être cité ; dans "Les plages entre réalité physique et représentation : état des lieux et résilience. Le cas des plages de Vias (GOLFE du lion) ». Il illustre un conflit entre les habitants de la commune et les services de l'état pour définir un plan d'action contre les érosions marines qui attaquent les plages.

\section{LA TABLE RONDE :}

Le colloque s'est terminé par une table ronde dont le titre était : "La résilience dans les risques. Une notion d'interface utile au décloisonnement, à la concertation, au partage des responsabilités?".
Le parti a été pris de réunir autour de cette table ronde, « des gens de terrain » plutôt que des chercheurs : Marc Fesneau, Député du Loir et Cher, Président du groupe Modem à l'Assemblée, ex maire de Marchenoir ; Philippe Bolo, Député du Maine et Loire (membre du bureau d'étude ISL) ; Pascal Mallet, Direction de l'information sur les risques majeurs, Communauté d'Agglomération Havraise ; Christian Piel, Urbaniste et Hydrologue, Directeur de l'agence Urbanwater (Paris) ; Regis Thépot, Directeur Général de l'EPTB (Etablissement Public Territorial de Bassin) Seine Grands Lacs.

\section{A la question de savoir si la notion de résilience leur semble utile et comprise :}

Marc Fesneau, fait part de son expérience de maire ayant vécu une inondation dans la commune dont il avait la charge. Il présente son rôle comme celui d'un coordonnateur veillant à ne pas ajouter du risque au risque (problèmes électriques). En dépit d'un certain découragement devant son impuissance face à l'inondation, la population a su se mobiliser pour faire face, se montrer capable de résilience.

Pascal Mallet témoigne du fait qu'au Havre, ville reconstruite après la guerre à une cote plus élevée qu'auparavant pour tenir compte du risque de submersion, la notion de résilience est très utile parce que mobilisatrice : on ne communique pas, dit-il, sur la réduction de la vulnérabilité, mais beaucoup mieux sur la résilience : l'intercommunalité du Havre travaille efficacement autour de cette notion.

Christian Piel fait part de son expérience d'urbaniste soucieux de redonner sa place à l'eau y compris dans les zones urbaines : nivellements, constructions sur pilotis mais aussi accompagnement au changement climatique avec le souci de végétalisation, de création d'îlots de fraicheur. Il considère que la question aujourd'hui n'est pas tant d'innover que d'associer les démarches (ex : habitat et environnement).

Régis Thépot considère la notion de résilience comme très positive et permettant une vision intégrée des problèmes. Il souligne toutefois la nécessité de se définir des objectifs clairs et prend pour exemple la crue de 2016 qui a touché la région parisienne : se définir pour objectif zéro dommages pour ce type de crue est accessible, ce type d'objectif clair et classique ne doit pas être oublié.

Philippe Bolo, par son métier d'ingénieur considère qu'un guide méthodologique sur " l'approche résilience » serait utile pour les Bureaux d'études.

\section{Quelle place donner au public dans les crises?}

Pascal Mallet note que les smartphones, les réseaux sociaux font beaucoup pour alerter mais qu'ils véhiculent aussi de fausses informations.

Marc Fesneau remarque que des comportements à risque sont encouragés parfois sur les réseaux sociaux. Il note l'importance de la continuité d'une parole publique pendant les crises.

Régis Thépot remarque qu'il y a lieu de faire bouger certains comportements. Il note que les PME et TPE ne sont pas assez sensibilisés aux risques et à la résilience : ils ont souvent reconstruit à l'identique après la crue de 2016.

\section{La résilience : un transfert de responsabilité de l'état vers les collectivités et les citoyens?}

La réponse est oui pour Régis Thépot qui donne l'exemple de la GEMAPI. Pascal Mallet parle de subsidiarité dans la réponse aux risques, de résilience à différentes échelles géographiques. Marc Fesneau considère la décentralisation 
comme étant d'une bonne logique mais qui ne doit pas entraîner un désintéressement de l'état. Christian Piel considère que la résilience est une question de gouvernance.

\section{CONCLUSIONS :}

La notion de résilience reste un sujet de débats. Pas tellement au niveau de sa définition qui se décline toujours au travers de l'idée de "faire face à un choc "*. Si la notion n'est pas toujours bien comprise par tout le monde, les témoignages de son intérêt sont nombreux, y compris parmi les acteurs de la gestion des risques, comme ont pu le montrer les débats à la table ronde.

Mais c'est au sujet des propriétés de la résilience que les débats restent compliqués. La notion de résilience est en effet largement considérée comme " intégratrice » (M. Reghezza, cf ci-dessus, et repris par beaucoup), ou " inclusive » (C. Cholez) ; elle permet de mieux prendre en considération les " échelles de l'espace d'action » (C. Cholez) et, par ailleurs, des responsables comme Pascal Mallet, Directeur de l'information sur les risques majeurs de la Communauté d'Agglomération Havraise, Christian Piel, Urbaniste et Hydrologue, Directeur de l'agence Urbanwater (Paris) et Regis Thepot, Directeur Général de l'EPTB (Etablissement Public Territorial de Bassin) Seine Grands Lacs reconnaissent son intérêt pratique. Pourtant la notion est aussi considérée par S. Ruffat comme représentant " un grand bond en arrière ». Pour d'autres, sans être aussi violemment contestée, elle reste néanmoins porteuse de «vertus quasi magiques »(cité par Corbillé), une notion « sale pour la science " (C. Cholez), "venue d'en haut, dans un contexte de réduction des engagements des Etats » (id). Elle est aussi interprétée comme un instrument un peu pervers, capable de justifier des contradictions (Pigeon). On a compris aussi que Régis Thépot, tout en lui reconnaissant la capacité à mieux prendre en charge la globalité des questions, note le danger de conduire à des objectifs manquant de clarté. Pour finir, sans chercher l'exhaustivité des critiques qui ont pu être formulées à l'encontre de la notion, il s'agirait, comme on l'a vu plus haut, d'un concept inspiré par un " référentiel néolibéral » (« reproche » formulé notamment par S. Rufat mais cité aussi par C. Morel-Journel et d'autres).

Un regard sur les interventions au colloque conduit pourtant à relever le fait que la notion a autorisé la prise en charge de la complexité des objets d'étude. Par exemple, les communications présentées au paragraphe 3 ci-dessus (Aide à la décision et résilience), s'intéressent à des réseaux d'alimentation en eau, des réseaux routiers, des sites industriels, des objets «villes ». Certes, ce n'est pas la première fois que ces objets complexes sont examinés du point de vue des risques mais, pour certains d'entre eux, le décloisonnement des types de risques, des objets d'étude, des analyses et diagnostics, des responsabilités (etc.) constitue une réelle avancée. Même si elle n'est pas toujours suffisante, la pluri

\footnotetext{
*. Ainsi, au travers des exposés, ont été relevées les définitions suivantes (pour n'en citer que quelques-unes) : "Aptitude à faire face à un choc " (Marc Vuillet) ; " Capacité d'organisation collective pour faire face à une inondation et en limiter les effets » (F. Rival) ; « La résilience urbaine est la capacité des personnes, communautés, institutions, entreprises et systèmes au sein d'une ville à survivre, s'adapter et se développer quels que soient les types de stress chroniques et de crises aiguës qu'ils subissent » (définition retenue dans le cadre des 100 Resilient Cities, citée par Sébastien Maire et Yona Jebrak). L'une des plus adaptée à notre contexte est sans doute celle-ci : « Capacité d'un territoire donné à faire face à des perturbations en mobilisant des mécanismes d'ajustement naturels et sociétaux pour se transformer et durer » (citée par Virginie Duvat).
}

et l'interdisciplinarité apparaissent facilitées par l'adoption de cette notion qui permet d'élargir les points de vue.

Plus encore, la notion de résilience associée aux risques oblige à faire référence à celle des territoires impactés. On a vu que de nombreuses communications citées en particulier au paragraphe 3 (L'indispensable échelle du « territoire »), mais aussi au paragraphe 5 (Résilience, concertation, gouvernance), situent bien les risques au cœur des territoires. Les territoires et leurs fonctionnements sont de véritables sujets d'étude et deviennent centraux pour l'analyse de la résilience. Le contexte (géographique, économique...), les relations entre les acteurs, l'histoire d'un territoire, sa gouvernance ... sont les déterminants d'une approche de la gestion des risques par la résilience. A cet égard, les communications de Bouisset et al. ("L'approche territoriale: Une condition de l'apport de la résilience à la prévention des désastres. Exemple du bassin industriel de Lacq face à ses futurs incertains ») et de Morel-Journel et al. (« La pollution industrielle des sols en héritage. De l'indifférence à la résilience? ») expriment bien les vraies limites de la notion de résilience dans les risques : Lorsque l'équilibre d'un territoire ne fait pas consensus (cas du bassin industriel de Lacq) ou lorsque les problèmes du territoire sont occultés, volontairement ou non, consciemment ou non, (cas des sols pollués de la région de St-Etienne) que signifie « faire face et se reconstruire »? Reproduire les mêmes imperfections ? La question, telle qu'elle est formulée par C. Cholez (et, en des termes voisins par M. Reghezza, S. Rufat et d'autres) est en effet la suivante : que faut-il préserver ? Qu'est-ce qui compte? Quelle est la «bonne situation »? Ces deux exemples d'une résilience introuvable relèvent de territoires où la question de « Ce qui compte » ne fait pas l'unanimité, où la situation actuelle n'est pas « la bonne situation ». Se trouve ainsi posée la question suivante : la résilience est-elle une notion pertinente pour gérer les risques dans le cas d'un territoire où la gouvernance n'est pas véritablement démocratique ? Plus prosaïquement, la notion de « Buid-back better " est-elle efficiente dans le cas de territoires industriels où il peut faire bon travailler mais pas vraiment vivre ?...

Pour finir, il semble que, en dépit de son caractère « flou », la notion de résilience soit intervenue sur la scène des risques à un moment propice pour aider à décloisonner les approches de la gestion des risques, pour mettre en dialogue les acteurs, s'interroger sur ce que sont les questions importantes pour les territoires. C'est ce qu'exprime clairement C. Cholez en conclusion de l'analyse qu'elle a menée : « ...mobiliser la notion de résilience peut, selon les contextes, permettre de questionner pratiquement les manières d'aborder les risques, d'organiser leur prise en charge et l'articulation des différents acteurs aux cultures professionnelles variées. En ce sens on peut parler d'effets concrets de la diffusion de cette notion. » Et aussi : «La dimension performative [de la résilience] tient sans doute à son ambivalence qui en fait une objet-frontière : sa flexibilité interprétative, la variation et la mise en relation des différentes échelles d'observation, de définition des problèmes et d'organisation, véhicule un changement de régime d'action/de posture ».

Ainsi, bien que sa définition ne lui permette pas d'acquérir le statut d'une grandeur mesurable, la résilience peut être créditée d'avoir créé du dialogue, permis de réinterroger la question de "ce qui compte ", d'avoir mis en avant la notion de territoire, avec tous ses aspects, y compris humains et sociaux. Il semble que la communauté des acteurs de la gestion des risques a bien compris ces enjeux. C'est en tout cas ce que donne à penser la tonalité des échanges durant le colloque et sa table ronde. 
Et maintenant ? Faisant retour sur le titre du colloque et notamment sur la question de « l'apport de la notion de résilience à la gestion des risques », que peut-on conclure ?

Dans sa lettre d'information (Méandre $\mathrm{N}^{\circ} 66$ de décembre 2017), Bruno Ledoux pose la question, après avoir rendu compte des actes du colloque. Il dit en effet "rester sur sa faim concernant les enseignements que les politiques publiques actuellement mises en æuvre, à l'échelle nationale comme à l'échelle locale, pourraient tirer de ces très nombreux travaux de recherche, ou bien comment les outils disponibles pourraient être revisités, améliorés, à partir de ces enseignements ». On a pu voir que plusieurs communications font état de traductions opérationnelles qui ont pu s'esquisser. Néanmoins, pour aller dans le même sens que B. Ledoux, il est clair que, entre la règle et son application, l'idée de résilience a introduit une forme de dialogue qu'il s'agit sans doute désormais de mieux formaliser et organiser. La mise en relation des différentes échelles d'action, l'articulation des différents acteurs, la question de « ce qui compte vraiment », etc. Toutes ces questions difficiles ont commencé à bouger grâce à la notion et de nouvelles initiatives sont nécessaires pour leur donner toute la place qu'elles méritent. Nul doute que le Ministère de l'Ecologie (MTES), à l'origine de l'appel à propositions de recherche sur le thème de la résilience, ne poursuive ces réflexions et intègre davantage la notion de résilience dans la politique de prévention.

\section{REMERCIEMENTS :}

La qualité du colloque et des échanges auxquels il a donné lieu doit beaucoup à celle de tous ses acteurs et actrices, à commencer par les membres de son comité de pilotage :
Stéphanie Bidault, Philippe Bolo, Anne Chanal, Thierry Coanus, Anna Dupont, Jean-Michel Grésillon, Michel Lang, Daniel Loudière, Didier Richard, Neda Sheibani, Dominique Thierry, Fredy Vinet ;

et de son conseil scientifique :

Marie Augendre, André Bachoc, Stéphanie Bidault, Anne Chanal, Thierry Coanus, Stéphane Friou, Jean-Michel Grésillon, Mehdi Ghoreychi, Michel Lang, Daniel Loudière, Céline Lutoff, Magalie Marty, Damienne Provitolo, Magalie Regheza, Anna Ribas, Didier Richard, Jean-Michel Tanguy, Freddy Vinet ;

qui ont contribué à en définir les objectifs, à analyser les propositions de communications, à animer les débats pendant le colloque.

Elle doit aussi et surtout à tous ceux qui sont intervenus dans ce colloque par leurs conférences (Magali Reghezza, Sébastien Maire), leurs animations (Patrice Buesot, Didier Richard, Damienne Provitolo, Freddy Vinet, Thierry Hubert), leurs communications, leurs posters et leurs questions.

Elle doit également beaucoup aux membres de la table ronde (Marc Fesneau, Philippe Bolo, Pascal Mallet, Christian Piel, Régis Thépot) qui ont exprimé le point de vue des professionnels et des responsables des collectivités sur la résilience, point de vue sans doute pas assez fortement présent au colloque.

Elle doit enfin à toute l'équipe de la Société Hydrotechnique de France qui a géré l'événement avec toutes ses difficultés administratives, informatiques, techniques ... Tout spécialement à Neda Sheibani qui a assuré l'essentiel de cette organisation.

Qu'ils en soient tous ici remerciés ! 